\title{
Construction of Sensory Transfer Model of Gustatory and Olfactory-Synaesthetic Metaphor (GO-STM) and English-Chinese Comparative Study
}

\author{
Hong Duan', Li Gao² \\ ${ }^{1}$ College of International Studies, Southwest University, Chongqing, China \\ ${ }^{2}$ Department of Foreign Languages, Northwest A\&F University, Yangling, China \\ Email: beyoungdh@163.com
}

Received 18 March 2014; revised 8 April 2014; accepted 15 April 2014

Copyright (C) 2014 by authors and Scientific Research Publishing Inc.

This work is licensed under the Creative Commons Attribution International License (CC BY). http://creativecommons.org/licenses/by/4.0/

\section{(c) (i) Open Access}

\section{Abstract}

This paper aims to explore the synaesthetic truth of taste and smell in particular. Based on the corpus of gustatory and olfactory-synaesthetic cases, classification and statistical tasks are undertaken, in which four main characters are discovered: 1) the particular sorts of gustatory and olfactory-synaesthetic metaphors; 2) the bi-directional transfer in specific pairs; 3) the hierarchical distribution among sensory modes; 4) the transfer frequencies of sensory transfer tendencies. Thus, GO-STM of both English and Chinese is constructed. What's more, the comparison of English and Chinese has been conducted for the first time. According to the statistic results, the percentage of English-Chinese gustatory and olfactory-synaesthetic dead metaphors is $48.1 \%$ and $48.3 \%$ respectively, and that's why we always ignore them. Furthermore, the embodiment basis of synaesthetic metaphors is analyzed in light of recent neurological research and Cognitive Linguistics.

\section{Keywords}

Synaesthesia, Gustatory and Olfactory Word, Sensory Transfer Model (STM), Gustatory and olfactory Sensory Transfer Model (GO-STM)

\section{Introduction}

Synaesthesia is metaphorical. It is a linguistic phenomenon and at the same time acts as a mode of thinking. In terms of English synaesthesia, for example, Ullmann (1957) and Williams (1976) have concluded the "hierar- 
chical distribution”, i.e., synaesthetic transfers from the "lower” to the "higher” sensory modes; Sean Day (1996) puts his interest in the connection of neurology and linguistics; with Chinese synaesthesia, for instance, Zhao Yanfang (2001) has also illustrated her viewpoint for synaesthesia; Meng Xiaoxi (2007) has designed her STM based on Chinese poetry. However, their researches lack in the detail illustration, sufficiency of data and discussion on gustatory and olfactory synaesthesia. What's more, up to now, there's no comparative study between English and Chinese in these two aspects. Based on previous studies, this thesis examines, refines and compares English-Chinese gustatory and olfactory-synaesthetic metaphors by means of data, and further puts forward Gustatory and Olfactory Sensory Transfer Model (GO-STM for short) with specific data distribution.

\section{Data Collection and Statistics}

\subsection{Data Collection and Description}

A major reason for compiling the corpus is to provide a basis for more accurate and reliable descriptions of how synaesthetic metaphors are structured and used. Large figures will therefore be needed to bring out any inherent regularity, and to reduce the margin of error to a minimum. In this study, ordinary language is chosen to the synaesthetic carriers, since they are too omnipresent to be ignored in daily life. The process of data collection is divided into three steps:

1) Word collection. To conduct the refinement, the choice of data is a big problem. In this thesis, Thesaurus (2003), Dictionary of Modern Chinese Classification (Dong, 1998) and Modern Chinese Dictionary (the 5th Edition), etc. are picked out as the source and standard of English-Chinese gustatory and olfactory words respectively, for as far as our knowledge is concerned, these dictionaries are the most authoritative in the field of Thesaurus. As the title of the thesis demonstrates, sense word is the scope of this study; accordingly, words of every part of speech listed in dictionaries are collected. As a result, 263 English gustatory words have been found, among which there are 128 nouns, 92 adjectives and 43 verbs, whereas, for English olfactory words, 129 have been found so far, in which there are 60 nouns, 43 adjectives and 26 verbs. On the other hand, altogether 98 Chinese gustatory words have been found, among which there are 11 nouns, 87 adjectives and as for the Chinese olfactory words, 41 have been collected, in which there are 5 nouns, 26 adjectives, 9 cross-class words (noun and adjective).

It seems that the number of words collected differs to a large extent in quantity, i.e., there are 263 English gustatory words, which is as three times as Chinese gustatory words; on the other hand, 129 English olfactory words have been collected, which is also three times more than Chinese olfactory words.

After a careful investigation, it is found that they differ greatly in the distribution of parts of speech. In English, almost every word has its own inflective forms, while for Chinese, it does not have inflective changes. Moreover, in English there are 64 words which refer to condiment or food with strong gustatory reflection, say, garlic, onion, etc., but the corresponding words haven't been listed in Dictionary of Modern Chinese Classification. Since these words are impossible to be synaesthetic, the corresponding Chinese expressions have not been looked up and listed.

2) Checking. To check out whether the collected words are synaesthetic or not, each of them is input into British National Corpus (BNC) for English and State Language Committee Corpus as well as Modern Chinese Corpus of Peking University for Chinese. As such, 45 English gustatory-synaesthetic words have been spotted, and all of them are adjectives. In addition, there are 7 English olfactory-synaesthetic words, and still all of them are adjectives. As for Chinese gustatory-synaesthetic words, the number is 62, in which there are 58 adjectives and 4 nouns. Compared with taste, olfactory-synaesthetic words are scant. There are only 4 , among which there are 3 adjectives and 1 verb.

3) Source confirmation. Synaesthesia, as a particular kind of metaphor, consists of two factors: the source sensory mode and the target sensory mode. Thus, to study the synaesthetic situation of gustatory and olfactory words, the source sensory mode should be traced back to get the appropriate transfer modes, i.e. which sense did the word originally describe? In this study, Ciyuan (2004), Cihai (2004), Haidian ${ }^{1}$, Dictionary.com ${ }^{2}$, Online Etymology Dictionary ${ }^{3}$ and Middle English Dictionary ${ }^{4}$ are applied as the referential standard for the source

\footnotetext{
${ }^{1}$ http://www.zdic.net/.

${ }^{2}$ http://dictionary.reference.com/.

${ }^{3}$ http://www.etymonline.com/.

${ }^{4}$ http://quod.lib.umich.edu/m/med/.
} 
sensory mode of each word which satisfies the requirement of this study. Furthermore, if the synaesthetic meaning has been listed in dictionary, viz., it will be marked as dead metaphors according to Modern Chinese Dictionary (Academy of Social Sciences Institute, 2005) and Oxford Advanced Learner's Dictionary (2005).

As thus, we have compiled complete lists of their 197 English-Chinese gustatory and olfactory-synaesthetic cases in which there are 81 for English and 116 for Chinese. Moreover, the number of English-Chinese gustatory and olfactory-synaesthetic dead metaphors is 39 and 56 respectively, taking up $48.1 \%$ and $48.3 \%$ of the total respectively.

\subsection{Methodology and Data Statistics}

\subsubsection{Methodology}

In order to demonstrate how the statistic work will be carried out, sample entries from "sweet" are taken for an example:

1) Translation, to modern English. Erm, Erm, through its virtues and sweet smell. No, no, sorry. Do it word for word. We

2) Lollipop. She would listen to me reading Coleridge, black hair falling over her sweet face, red boots at the side of the bed. TRYING TO BEGIN He

3) From the cot, and was cuddling him softly, singing in a tiny, sweet voice to him till he quietened. Bridhe stood still, afraid to move (as cited from BNC).

Thus, we have "sweet smell", "sweet face", "sweet voice". They would be recorded into its appropriate spread-sheet as follows in Table 1.

We then record these into a calculation table, as Table 2 follows.

The senses being talked about, or "primary senses", are listed vertically in the left-hand column; the secondary, synaesthetic senses into which the terms are placed metaphorically are listed horizontally across. Thus, for example, in the upmost right-hand corner is "touch in terms of sound", as in "soft music". Sums for each row and column are given, respectively, on the right-hand side for the mode in which the primary sense is placed. The dotted line running diagonally across falls where a sense is talked about in terms of itself; these will always remain zero, as such phrases are not synaesthetic metaphors.

We then add all numbers in the vertical column for a particular sense and subtract from that number the sum of the numbers across a row for that same sense. In other words, we subtract the number of times that sense is used metaphorically to talk about other senses from the times a particular primary sense is talked about. Using this algorithm, if synaesthetic metaphors are random and evenly distributed, all of these sums would be zero across the board. As they are not, the negative/positive value indicates the weighing of a sense in a "ranking" sequence. The senses are placed in order from greatest positive value to greatest negative value, which reflects the sequence of least marked sense to most marked sense. Table 3 reflects this ranking for the three examples from "sweet".

\subsubsection{Data Statistics}

In this section, the statistic findings of both English-Chinese gustatory and olfactory-synaesthetic metaphor will be demonstrated by means of the approach as Section 2.2.1 illustrates. With an attempt to investigate only the gustatory and olfactory-synaesthetic metaphors, the input and output routes pertaining to the two senses will be focused, viz., we will just compile the gustatory and olfactory-synaesthetic metaphors of those input and output transfers to and from the other four sensory modes. Therefore, such pairings will never be seen in this study as:

$$
\begin{aligned}
& \text { touch } \rightarrow \text { vision } \\
& \text { touch } \rightarrow \text { sound } \\
& \text { vision } \rightarrow \text { touch } \\
& \text { vision } \rightarrow \text { sound } \\
& \text { sound } \rightarrow \text { touch } \\
& \text { sound } \rightarrow \text { vision }
\end{aligned}
$$

1) Corpus of Gustatory and Olfactory-Synaesthetic Metaphors in English

Following the way of Section 2.2.1, a corpus of gustatory and olfactory-synaesthetic transfer model in English is harvested as Table 4 shows.

The ranking for this data is as per Table 5. 
Table 1. Sample entries from "sweet”.

\begin{tabular}{rr}
\hline taste $\rightarrow$ smell & sweet smell \\
\hline taste $\rightarrow$ vision & sweet face \\
taste $\rightarrow$ sound & sweet voice \\
\hline
\end{tabular}

Table 2. Sample spread-sheet for the tabulation of three entries from "sweet".

\begin{tabular}{ccccccc}
\hline \multirow{2}{*}{$\begin{array}{c}\text { Primary } \\
\text { Senses }\end{array}$} & Touch & Taste & Smell & Vision & Sound & Total \\
\cline { 2 - 6 } Touch & - & 0 & 0 & 0 & 0 & 0 \\
Taste & 0 & - & 1 & 1 & 0 & 3 \\
Smell & 0 & 0 & - & 0 & - & 0 \\
Vision & 0 & 0 & 0 & 0 & - & 0 \\
Sound & 0 & 0 & 0 & 1 & 1 & 3 \\
Total & 0 & 0 & 1 & & 0 \\
\hline
\end{tabular}

Table 3. Ranking of three sample examples from "sweet”.

\begin{tabular}{ccc}
\hline & Secondary Minus Primary & Ranking \\
\hline Taste & $(3-0)$ & 3 \\
Touch & $(0-0)$ & 0 \\
Smell & $(0-1)$ & -1 \\
Vision & $(0-1)$ & -1 \\
Sound & $(0-1)$ & -1 \\
\hline
\end{tabular}

Table 4. Total data of gustatory and olfactory-synaesthetic metaphors in English.

\begin{tabular}{ccccccc}
\hline \multirow{2}{*}{$\begin{array}{c}\text { Primary } \\
\text { Senses }\end{array}$} & Touch & Taste & Smell & Vision & Sound & Total \\
\cline { 2 - 7 } Touch & - & $\mathbf{9}$ & $\mathbf{5}$ & 0 & 0 & 14 \\
Taste & $\mathbf{3}$ & - & $\mathbf{1 4}$ & $\mathbf{1 2}$ & $\mathbf{1 1}$ & $\mathbf{4 0}$ \\
Smell & $\mathbf{1}$ & $\mathbf{2}$ & - & $\mathbf{4}$ & $\mathbf{2}$ & $\mathbf{9}$ \\
Vision & 0 & $\mathbf{1 2}$ & $\mathbf{3}$ & $\mathbf{1 2}$ & $\mathbf{0}$ & 3 \\
Sound & 0 & $\mathbf{2}$ & $\mathbf{1}$ & 0 & - & 13 \\
Total & 4 & $\mathbf{2 5}$ & & 16 & $\mathbf{8 1}$ \\
\hline
\end{tabular}

As Table 5 demonstrates, it is found that smell outstrips the other senses as the most common for which to attach metaphors; taste are the most common sense are expressed in.

The percentages for the total data of gustatory and olfactory-synaesthetic metaphors in English are as follows in Table 6.

Table 6 gives us the following information:

a) In English corpus of gustatory and olfactory-synaesthetic metaphors, the theoretically predicted 14 patterns of transfer routes have been found.

b) Among the 81 cases, there are 52 cases in accordance with the "hierarchical distribution" law, which takes up $64.2 \%$ of the total. That is to say, this study reinforces Ullmann’s claim soundly. 
Table 5. Ranking for the complete data of gustatory and olfactory-synaesthetic metaphors in English.

\begin{tabular}{ccc}
\hline & Secondary Minus Primary & Ranking \\
\hline Taste & $(40-25)$ & 15 \\
Touch & $(14-4)$ & 10 \\
Vision & $(15-16)$ & -1 \\
Sound & $(3-13)$ & -10 \\
Smell & $(9-23)$ & -14 \\
\hline
\end{tabular}

Table 6. Rates of occurance for gustatory and olfactory-synaesthetic metaphors in English.

\begin{tabular}{ccc}
\hline Type of Metaphor & Rate (\%) \\
\hline taste $\rightarrow$ smell & $17.3 \%$ \\
taste $\rightarrow$ vision & $14.8 \%$ \\
vision $\rightarrow$ taste & $14.8 \%$ \\
taste $\rightarrow$ sound & $13.6 \%$ \\
touch $\rightarrow$ taste & $11.1 \%$ \\
touch $\rightarrow$ smell & $6.2 \%$ \\
smell $\rightarrow$ vision & $4.9 \%$ \\
vision $\rightarrow$ smell & $3.7 \%$ \\
taste $\rightarrow$ touch & $3.7 \%$ \\
smell $\rightarrow$ taste & $2.5 \%$ \\
smell $\rightarrow$ sound & $2.5 \%$ \\
sound $\rightarrow$ smell & $2.5 \%$ \\
smell $\rightarrow$ touch & $1.2 \%$ \\
sound $\rightarrow$ smell & $1.2 \%$ \\
\hline Total & $100.0 \%$ \\
\hline
\end{tabular}

c) Although there exists a large proportion of regular cases, it also reminds us that the other overwhelming number of irregular cases, viz., 35.8\% prevails as well.

2) Corpus of Gustatory and Olfactory-Synaesthetic Metaphors in Chinese

Following the same fashion, the corpus of gustatory and olfactory-synaesthetic metaphors in Chinese is constructed as Table 7 shows.

The ranking for this data is as per Table 8 .

As Table 8 demonstrates, it is found that smell as well as sound outstrips the other senses as the most common for which to attach metaphors; taste are the most common sense are expressed in.

The percentages for the total data of olfactory-synaesthetic metaphors in Chinese are as follows in Table 9 .

Table 9 gives us the following information:

a) In Chinese corpus of gustatory and olfactory-synaesthetic metaphors, the theoretically predicted 13 patterns of transfer routes have been found with "smell $\rightarrow$ touch" excluded.

b) Among the 116 cases, there are 82 cases in accordance with the "hierarchical distribution" law, which takes up $70.7 \%$ of the total. That is to say, this study abides by Ullmann's claim soundly again.

c) Although there exists a large proportion of regular cases, it also reminds us that the other overwhelming number of irregular cases, viz., 29.3\% prevails as well. 
Table 7. Total data of gustatory and olfactory-synaesthetic metaphors in Chinese.

\begin{tabular}{ccccccc}
\hline \multirow{2}{*}{$\begin{array}{c}\text { Primary } \\
\text { Senses }\end{array}$} & Touch & Taste & Smell & Vision & Sound & Total \\
\cline { 2 - 7 } Touch & - & $\mathbf{1}$ & $\mathbf{1}$ & 0 & 0 & 2 \\
Taste & $\mathbf{1}$ & - & $\mathbf{2 8}$ & $\mathbf{2 0}$ & $\mathbf{2 4}$ & $\mathbf{7 3}$ \\
Smell & $\mathbf{0}$ & $\mathbf{8}$ & - & $\mathbf{5}$ & $\mathbf{3}$ & $\mathbf{1 6}$ \\
Vision & 0 & $\mathbf{1 3}$ & $\mathbf{6}$ & - & 0 & 19 \\
Sound & 0 & $\mathbf{4}$ & $\mathbf{2}$ & 0 & - & 6 \\
Total & 1 & $\mathbf{2 6}$ & $\mathbf{3 7}$ & 25 & 27 & $\mathbf{1 1 6}$ \\
\hline
\end{tabular}

Table 8. Ranking for the complete data of gustatory and olfactory-synaesthetic metaphors in Chinese.

\begin{tabular}{ccc}
\hline & Secondary Minus Primary & Ranking \\
\hline Taste & $(73-26)$ & 47 \\
Touch & $(2-1)$ & 1 \\
Vision & $(19-25)$ & -7 \\
Sound & $(16-37)$ & -21 \\
Smell & $(6-27)$ & -21 \\
\hline
\end{tabular}

Table 9. Rates of occurance for gustatory and olfactory-synaesthetic metaphors in Chinese.

\begin{tabular}{cc}
\hline Type of Metaphor & Rate (\%) \\
\hline taste $\rightarrow$ smell & $24.1 \%$ \\
taste $\rightarrow$ sound & $20.7 \%$ \\
taste $\rightarrow$ vision & $17.2 \%$ \\
vision $\rightarrow$ taste & $11.2 \%$ \\
smell $\rightarrow$ taste & $6.9 \%$ \\
vision $\rightarrow$ smell & $5.2 \%$ \\
smell $\rightarrow$ vision & $4.3 \%$ \\
sound $\rightarrow$ taste & $3.4 \%$ \\
smell $\rightarrow$ sound & $2.6 \%$ \\
sound $\rightarrow$ smell & $1.7 \%$ \\
touch $\rightarrow$ taste & $0.9 \%$ \\
touch $\rightarrow$ smell & $0.9 \%$ \\
taste $\rightarrow$ touch & $0.9 \%$ \\
\hline Total & $100.0 \%$ \\
\hline
\end{tabular}

\section{The Construction and Comparison of GO-STM}

\subsection{Characters of GO-STM}

From Section 2.2.2, at least four points can be concluded: 1) the categories of sensory transfer tendency; 2) the hierarchical relation among sensory modes; 3) the bi-directional transfer between specific pair of sensory modes; 4) the frequencies of each transfer categories. 


\subsubsection{The Particular Sorts of Gustatory and Olfactory-Synaesthetic Metaphors}

In former models, a sort of synaesthetic metaphor is illustrated by an arrow, which stands for a kind of transfer tendency between two modes, arrowhead denoting transfer tendency. Ullmann's linear model has five arrows pointing at the same direction, from the lower to the higher reaches of the sensation. That is to say, Ullmann (1957) suggests 5 sorts of synaesthetic metaphors, namely 5 transfer tendencies, but he does not discuss the transfer category in detail. Williams (1976) offers a model with 9 arrows, some of which mount from the same mode to different ones, i.e. one sensory mode can act as the source domain in several transfer tendencies. With taste and smell, Williams suggests only 3 kinds of transfer pattern, i.e., taste $\rightarrow$ smell, taste $\rightarrow$ sound, and touch $\rightarrow$ taste. Following Ullmann (1957), Sean Day (1996) employs linear figure containing identical sensory mode with little difference in order. Yu Ning (2003) employs "dimension" and "color" instead of vision, since in his opinion the former two perceptions are the primary factors constituting the latter one. Furthermore, Zhao Yanfang (2001) develops the category to seven sensory modes and proposes 4 sorts of gustatory and olfactory-synaesthetic metaphors. Meng (2007) offers a model with 6 transfer routes.

This thesis employs traditional means of mode classification to make the comparative results clearer. After investigating the corpus of 81 gustatory and olfactory-synaesthetic metaphors in English, the theoretically prospective 14 sorts of synaesthetic transfer have been found. After probing the corpus of 116 gustatory and olfactory-synaesthetic metaphors in Chinese, there are 13 sorts of synaesthetic transfer which have been found with only one pairing, viz., smell $\rightarrow$ touch excluded.

\subsubsection{The Bi-Directional Transfer in Specific Pairs}

Ullmann finds that numerical evidence indicates a general trend of movement: transfers tend to mount from the lower to the higher stages of the sensation, but not vice versa. Then, he builds a linear model. Other scholars develop his model based on their corpus, but merely modify or add some modes, and particularize more sorts of transfer tendencies. Their models contain arrows heading one direction. According to the corpus collected here, we have found "irregularity" cases, i.e. some cases with transfer tendency of the opposite direction.

In Table 4, Table 6, Table 7 and Table 9, it is easy to discover the bi-directional transfer in specific pairs. Bi-directional transfers exist in those pairs of sensory modes, namely the pairs of taste vs touch, taste vs smell, taste vs vision, taste vs sound, smell vs touch, smell vs vision, and smell vs sound. Therefore, the arrows standing for the above transfer tendency should have two heads.

\subsubsection{The Hierarchical Distribution among Sensory Modes}

Ullmann is the first who finds the general trend of movement. And then, several scholars validate the conclusion about hierarchical distribution of sensory modes. All their models, both linear and rectangular ones, set the order of modes following the hierarchical distribution.

As that fact can be found, when GO-STM is constructed, the hierarchical distribution will be followed, like Figure 1 shows.

\subsubsection{The Transfer Frequencies of Sensory Transfer Tendencies}

The former models have merely indicated both mode categories and their transfer tendencies, but never mentioned the transfer frequency of each tendency. Based on corpus analysis, the fact is found that each tendency does not happen with the same frequency.

\subsection{Comparison between English and Chinese Based on GO-STM}

\subsubsection{Similarities and Differences between the Two GO-STMs}

In order to make the comparison direct, the gross GO-STM of English-Chinese gustatory and olfactory-synaesthetic metaphors is constructed as Figure 2 shows.

As Figure 2 demonstrates, the gustatory and olfactory-synaesthetic mechanism is similar between English and Chinese with slight difference in specific linguistic expressions and frequency in terms of the following points:

1) English and Chinese GO-STMs are similar in transfer pattern to some degree. In English GO-STM, 14 theoretically possible transfer routes have been found; in Chinese GO-STM, 13 patterns have been spot. That is to say, the two models do share the same 13 transfer routes. However, there is one pairing, "smell $\rightarrow$ touch", which has not yet been seen in 116 instances of Chinese gustatory and olfactory-synaesthetic metaphor. 


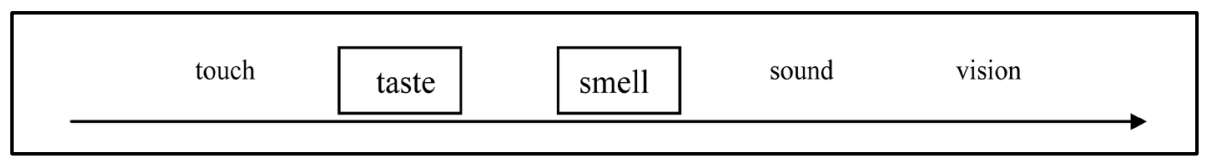

Figure 1. Sequence of sensory modes in English GO-STM.

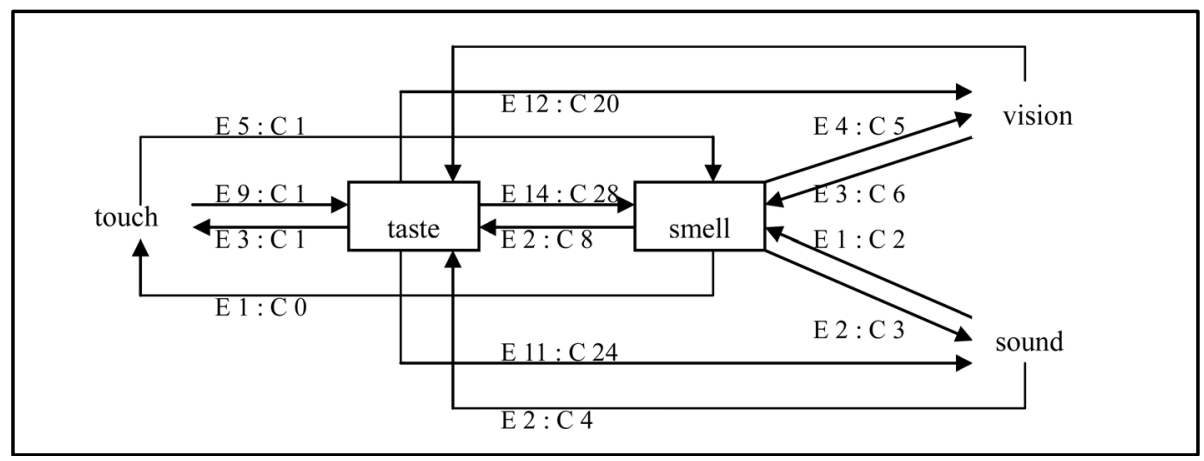

(note: $\mathrm{E}=$ English; $\mathrm{C}$ = Chinese).

Figure 2. Gross GO-STM of English-Chinese gustatory and olfactory-synaesthetic metaphors.

2) No matter English or Chinese GO-STM, there is a very large proportion of transfer routes, which conforms to the hierarchical distribution, i.e., transfers tend to mount from the lower to the higher reaches of the sensation, from the less differentiated sensations to the more differentiated ones. With respect to English, there is 64.2\% regular transfer pattern, while as for Chinese, there is $70.7 \%$. The statistic results reinforce the conclusion of the previous researches.

3) In spite of the overwhelming percentage of regular transfer routes, the frequency of cases violating the "low to high" law is disproportionally under-represented. With English GO-STM, there is 35.8\%, while for Chinese GO-STM, there is $29.3 \%$. The very proportion is too large to be ignored.

4) In both English and Chinese GO-STM, taste tends to transfer hierarchically to smell, vision and sound and receive transfers from the sense of touch. Smell is apt to transfer hierarchically to vision, sound and receive transfers from the sense of touch and taste. The specific percentage can be seen as Table 10 shows.

Table 10 also indicates that taste and smell can violate the "low to high" law. What's more, the number of "high to low" transfer routes is remarkable. In English GO-STM, taste is expected to transfer back to the lower sensation, touch, and receive transfers from the higher sensation, viz., smell, vision and sound; smell is expected to transfer back to the lower sensation, touch and taste, and receive transfers back from the higher sensation, vision and sound. On the contrary, in Chinese GO-STM, taste may transfer back to the lower sensation, touch, and receive transfers from the higher sensation, smell, vision and sound; smell is likely to transfer back to the lower sensation of taste expect for touch and receive transfers from the higher sensation of vision and sound. As for the non-existence of "smell $\rightarrow$ touch" pairing, it is perhaps because of the paucity of statistics.

5) As the Section 2.1 indicates, in English, 39 gustatory and olfactory-synaesthetic dead metaphors have been found, which take up $48.1 \%$ of the total. On the contrary, in Chinese, 56 gustatory and olfactory-synaesthetic dead metaphors have been spot, which account for $48.3 \%$ of the total. These dead metaphors are entrenched in our daily life so that we even do not realize they are metaphorical. However, in this study, there are $64.2 \%$ regular transfer routes in English GO-STM and 70.7\% in Chinese one. Therefore, we can predict that the other regular synaesthetic meaning is probably to be added in dictionary in the near future.

\subsubsection{Reasons for the Similarities and Differences}

1) Reasons for the Similarities

According to Lakoff \& Johnson (1999: p. 20), “an embodied concept is a neural structure that is actually part of, or makes use of, the sensorimotor system of our brains. Much of conceptual inference is, therefore, sensorimotor inference.” Our conceptual system is grounded in, naturally makes use of, and is crucially shaped by our conceptual and motor systems. We can only form concepts through the body. "Therefore, every understanding of the world or ourselves is framed in terms of concepts shaped by our bodies” (Lakoff \& Johnson, 1999: p. 555). 
Table 10. Percentage of gustatory and olfactory-synaesthetic metaphors observing hierarchy distribution.

\begin{tabular}{ccccc}
\hline \multirow{2}{*}{ Category } & \multicolumn{3}{c}{ English } & \multicolumn{2}{c}{ Chinese } \\
\cline { 2 - 5 } & Gustatory words & Olfactory words & Gustatory words & Olfactory words \\
\hline Percentage & $69.2 \%$ & $91.0 \%$ & $73.8 \%$ & $69.8 \%$ \\
\hline
\end{tabular}

Since human enjoys the same body structure and live in the same environment, our perceptions tend to be similar. Therefore, English GO-STM and Chinese GO-STM share the same 13 synaesthetic transfer routes.

Apart from that, synaesthetic metaphors are embodied in two senses. Firstly, recent neurological research suggests that the executive areas of the human brains, primary in the front lobes, manifest a high degree of sensory integration, which means neural connections between five senses are possible. Therefore, synaesthetic metaphors may be a manifestation of this sensorial interaction. Secondly, human beings tend to use basic-level concepts to characterize concepts that are more abstract.

For example, we use terms of our bodily experience, say, taste and smell to express more complicated concepts, like "bitter smile" and "redolent face", etc. As concept and reason both derive from the sensorimotor system, the mind could not separate from or independent of the body.

In addition, as the rapid growth of research interest on theories of multisensory integration, "flavor" tends to be used as a term to describe the combination of tastes, smells, trigeminal, and tactile sensations as well as the visual and auditory cues, that we perceive when eating food, which "may be indicative of the fact that the taxonomy currently used to define our senses is simply not appropriate" (Auvray \& Spence, 2008: p. 1016). The fruits of the latest neurology can provide sound evidence for the statistic results and GO-STM proposed in this study. The idea of a functional flavor perceptual system which includes both smell and taste is actually anticipated many years ago by Brillat-Savarin (1835: p. 41) who wrote that he was "tempted to believe that smell and taste are in fact but a single sense, whose laboratory is in the mouth and those chimney is the nose". This conception of flavor as a perceptual system has also been proposed by Gibson (1966) under the term "tasting system" which additionally includes the sense of touch. The different receptors for the volatile and the soluble components of food can be incorporated into the same perceptual system. Furthermore, this perceptual system should also include the haptic components associated with the act of eating. As one of the most important functions of the mouth is testing substances for their palatability, the tongue and the oral cavity are sensitive to size, shape, texture, consistency, and temperature. Indeed, although we usually think of the mouth as an entirely different organ belonging to the different sensory modalities, it actually overlaps with the tactile/haptic system. Thus, when drinking or eating, we experience a multitude of sensations, including taste and smell, but also touch, temperature, and sometimes pain/irritation in the oral cavity and/or nose.

The tasting system is conceived of by Gibson (1966) basically as a system for the control of ingestion. The pick-up of all the available information about a substance in the mouth cuts across the ordinary classification of different receptors types. It includes two types of the tongue and nearby tissue lining the mouth (the usual sense of taste). The volatile (odorous) component of the substance stimulates the receptors in the olfactory cavity above the mouth (via retronasal olfaction). This perceptual system also includes the receptors for oral haptic information, which are located in the skin and tissue of the tongue, in the lips, in the lining of the mouth, in the muscles of the tongue and in the joints of the jaw. The surface texture of the substance is registered by a sort of mouth palpation, which detects such properties as slipperiness, smoothness, and roughness. The consistency of the substance is registered by chewing, which detects such properties as viscosity, elasticity, and other tactile qualities such as softness, hardness or brittleness.

It should be noted that the flavor system can be conceiced of either as being posited on the basis of sensations, or on the basis of the extraction of information. For instance, nearly a century ago, Tichener also emphasized the curiously unitary character of the combined sensations of taste, smell, touch, and temperature when eating a peach or drinking a cup of coffee.

Scientific experimentations prove that human beings experience synaesthesia due to their inherent physiological and psychological mechanism, in other words, synaesthesia is the actual experience to some extent in daily life. As one of essential viewpoints held by cognitive linguistics, the embodiment of language suggests that language derives from interaction between subjectivity and objectivity, as the result of the interaction between human beings and the external world, grounded on cognition. Therefore, synaesthetic metaphor of language is based on synaesthesia experience in daily life. 
The physiology and the latest fruits of neurological studies mentioned above are in accordance with the statistic findings. Firstly, the sequence of sensory modes is as the same as the sequence of time in which different sensory organs reach mature in time order.

Secondly, the close relationship of touch, taste, smell, and vision, etc. has been sounded loudly to create another term "flavor" to indicate the multisensory integration among them. With the further development of neurology, we may find more support for our research results.

2) Reasons for the Differences

The most fundamental values in a culture will be coherent with the metaphorical structure of the most fundamental concepts in the culture (Lakoff \& Johnson, 1980: p. 22). As conceptual metaphor is grounded in a variety of human experiences that are universal at the generic level across different cultures, metaphor acquires its universality across different cultures. Another term that goes hand in hand with the cultural universality is cultural variation, that is, metaphor varies in different cultures. Kovecses (2002: p. 165) suggests the following possibilities for cultural variation for metaphors:

a) Variation in the range of conceptual metaphors for a given target;

b) Variation in the particular elaborations of conceptual metaphors for a given target.

The first case indicates that there can be differences in the range of conceptual metaphors that languages and cultures have available for the conceptualization of particular target domains. The second case indicates that two languages may share the same conceptual metaphor, but the metaphor will be elaborated differently in the two languages.

Although the reactions of human sense organs to the same stimulation are not different due to the differences in nationality, the associations by similarity arises by the same stimulation from outside are not always the same.

Take the gustatory word "suan" (somewhat like sour in English) in Chinese for an example, some words may be felt "suan liu liu" (sour) when heard by people, thus we have "suan liu liu de shuo zhe". Here taste mixes sound, and "suan liu liu" may mean jealousy, ironic or something like that. However, in English, there's no relation between jealousy and sour. The word "sour" has another kind of association of synaesthesia in English. When it is related to vision, it usually means angry, ferocious, or hostile, for example, "to have a sour smile" and so forth.

It seems that the differences of synaesthetic association can only be due to the differences of national cultures. The differences are also called "the common inherited scheme of conception of a nation" by F. M. Cornford. It is just like the air surrounding us and controlling our thought activities in various ways. Although synaethesia is based on the similarities of human physiology, it is still influenced by "the common inherited scheme of conception of a nation".

\section{Conclusion}

This study is the first pilot study, which aims to explore the synaesthetic truth of taste and smell in particular. Based on the corpus of gustatory and olfactory-synaesthetic cases, classification and statistical tasks are undertaken. Four main characters are discovered: 1) particular sorts of synaesthetic metaphors; 2) the bi-directional transfer in specific pairs; 3) the hierarchical distribution among sensory modes; 4) the transfer frequencies of sensory transfer tendencies. Thus, GO-STM of both English and Chinese is constructed, which not only inherits achievements held by the previous model, but also fills the gap left by previous studies. What's more, the comparison of English and Chinese has been conducted for the first time. Moreover, according to the statistic results, the percentage of English-Chinese gustatory and olfactory-synaesthetic dead metaphors is $48.1 \%$ and $48.3 \%$ respectively, and that's why we always ignore them. Furthermore, the embodiment basis of synaesthetic metaphors is analyzed in light of recent neurological research and Cognitive Linguistics.

\section{References}

Academy of Social Sciences Institute (2005). Modern Chinese Dictionary (5th ed.). Beijing: The Commercial Press.

Auvray, M., \& Spence, C. (2008). The Multisensory Perception of Flavor. Consciousness and Cognition, 17, $1016-1031$. http://dx.doi.org/10.1016/j.concog.2007.06.005

Brillat-Savarin, J. A. (1835). The Physiology of Taste. In A Handbook of Gastronomy. London: Nimmo \& Bain.

Cihai (2004). Cihai. Beijing: The Commercial Press.

Ciyuan (2004). Ciyuan. Beijing: The Commercial Press. 
Dong, D. N. (1998). Dictionary of Modern Chinese Classification. Shanghai: Chinese Dictionary Press.

Day, S. (1996). Synaesthesia and synaesthetic metaphors. Psyche, 2, 1-16.

Gibson, J. J. (1966). The Senses Considered as Perceptual Systems. Boston, MA: Houghton Mifflin.

Kovecses, Z. (2002). Metaphor. London: Oxford University Press.

Lakoff, G., \& Johnson, M. (1980). Metaphors We Live by. Chicago and London: University of Chicago Press.

Lakoff, G., \& Johnson, M. (1999). Philosophy in the Flesh: The Embodied Mind and Its Challenge to Western Thought. New York: Basic Books.

Meng, X. X. (2007). Construction of Sensory Transfer Modal (STM) in Synaesthetic Metaphor-A Study Based on the Corpus of Chinese Poetry. MA Thesis, Chongqing: Sichuan International Studies University.

Oxford Advanced Learner's Dictionary (2005). Oxford Advanced Learner's Dictionary. London: Oxford University Press.

Ullmann, S. (1957). The Principles of Semantics. Oxford: Blackwell.

Williams, J. (1976). Synaesthetic Adjectives: A Possible Law of Semantic Change. Language, 52, 461-478. http://dx.doi.org/10.2307/412571

Yu, N. (2003). Synesthetic Metaphor: A Cognitive Perspective. Journal of Literary Semantics, 32, 19-34. http://dx.doi.org/10.1515/jlse.2003.001

Zhao, Y. F. (2001). An Introduction to Cognitive Linguistics. Shanghai: Shanghai Foreign Language Education Press. 